\title{
Psychological, behavioral and social effects of disclosing Alzheimer's disease biomarkers to research participants: a systematic review
}

\author{
S. A. S. A. Bemelmans', K. Tromp², E. M. Bunnik², R. J. Milne³, S. Badger ${ }^{3}$, C. Brayne', M. H. Schermer²
} and E. Richard ${ }^{1 *}$

\begin{abstract}
Background: Current Alzheimer's disease (AD) research initiatives focus on cognitively healthy individuals with biomarkers that are associated with the development of $\mathrm{AD}$. It is unclear whether biomarker results should be returned to research participants and what the psychological, behavioral and social effects of disclosure are. This systematic review therefore examines the psychological, behavioral and social effects of disclosing genetic and nongenetic AD-related biomarkers to cognitively healthy research participants.
\end{abstract}

Methods: We performed a systematic literature search in eight scientific databases. Three independent reviewers screened the identified records and selected relevant articles. Results extracted from the included articles were aggregated and presented per effect group.

Results: Fourteen studies met the inclusion criteria and were included in the data synthesis. None of the identified studies examined the effects of disclosing nongenetic biomarkers. All studies but one concerned the disclosure of APOE genotype and were conducted in the USA. Study populations consisted largely of cognitively healthy first-degree relatives of AD patients. In this group, disclosure of an increased risk was not associated with anxiety, depression or changes in perceived risk in relation to family history. Disclosure of an increased risk did lead to an increase in specific test-related distress levels, health-related behavior changes and long-term care insurance uptake and possibly diminished memory functioning.

Conclusion: In cognitively healthy research participants with a first-degree relative with $A D$, disclosure of APOE ع4-positivity does not lead to elevated anxiety and depression levels, but does increase test-related distress and results in behavior changes concerning insurance and health. We did not find studies reporting the effects of disclosing nongenetic biomarkers and only one study included people without a family history of AD. Empirical studies on the effects of disclosing nongenetic biomarkers and of disclosure to persons without a family history of AD are urgently needed.

Trial registration: PROSPERO international prospective register for systematic reviews CRD42016035388. Registered 19 February 2016.

Keywords: Alzheimer's disease, Biomarkers, Disclosure, Risk, Psychological effects, Behavioral effects, Social effects, Prevention studies, Clinical research, Ethics

\footnotetext{
* Correspondence: edo.richard@radboudumc.nl

'Department of Neurology, Radboudumc, Geert Grooteplein-Zuid 10, 6525

GA Nijmegen, The Netherlands

Full list of author information is available at the end of the article
} 


\section{Background}

Despite numerous studies conducted in the past two decades, there are currently no effective disease-modifying treatments or evidence-based preventive interventions for Alzheimer's disease (AD) [1]. Because the pathological processes underlying the development of clinical $\mathrm{AD}$ are thought to precede the onset of symptoms by years to decades [2-4], previous interventions aiming to prevent $\mathrm{AD}$ may have been initiated too late in the disease process. Recently, research initiatives have therefore turned to people who are cognitively healthy but supposedly at increased risk of developing $\mathrm{AD}$ on the basis of AD-related biomarkers, in order to slow down or halt the pathological processes and prevent the onset of clinical AD symptoms [5, 6]. Examples of these biomarkers are low amyloid beta 42 and high total or phosphorylated tau levels in cerebrospinal fluid (CSF) [7], positive amyloid PET scans [8] and genetic markers such as the apolipoprotein E (APOE) $\varepsilon 4$ genotype [9].

When biomarker examinations are performed in the context of research in people without cognitive complaints, the question arises of whether the results of these examinations should actively be returned to study participants.

Whether active disclosure of biomarker status should be pursued depends on the potential benefit but also potential harm it may cause to research participants. Of all health risks people face in life, many people in both Europe and the USA fear developing AD the most $[10,11]$. APOE genetic testing and nongenetic biomarkers such as brain amyloid imaging may indicate an increased risk of developing $\mathrm{AD}$, but do not predict with certainty if and when someone will develop clinical symptoms [12, 13]; the link between, for instance, amyloid positivity and cognitive decline remains particularly elusive [14-16]. Moreover, because there is no disease-modifying treatment or prevention strategy available, the possibility to act upon an increased risk of $\mathrm{AD}$ is limited. Based on these considerations, it is plausible that the disclosure of AD-related biomarkers is associated with an unfavorable balance of risks and benefits for some people.

Empirical evidence for the effects of AD genetic susceptibility testing has been reviewed previously $[17,18]$. Recent prevention studies do not select individuals solely on the basis of their genotype but also on the basis of nongenetic biomarkers $[5,6]$. The impact of disclosing these nongenetic results, which inform research participants of an ongoing pathological process in their brains, is potentially different from the impact of genotype disclosure, which informs of a risk. The effects of disclosing nongenetic AD biomarkers to cognitively healthy research participants have not been reviewed systematically. This systematic review therefore addresses the following question: what are the psychological, behavioral and social effects of disclosing genetic and nongenetic AD-related biomarkers to cognitively healthy research participants?

\section{Methods}

This systematic review is reported in accordance with the Preferred Reporting Items for Systematic Review and Meta-analyses (PRISMA) statement [19].

\section{Data sources and search strategy}

We conducted a systematic literature search in eight electronic databases on April 28, 2015: Embase, Medline, PsycINFO, Cumulative Index to Nursing and Allied Health Literature (CINAHL), Cochrane Central Register of Controlled Trials, Web of Science, PubMed and Google Scholar. The search strategy was developed in collaboration with an information specialist from the Erasmus Medical Centre Medical Library. Search terms used were variations on the key words: dementia, biological markers, genetic testing, disease risk, disclosure, and psychological, behavioral and social factors. The full search strategies for all databases are presented in Additional file 1. Reference lists of included articles were hand searched for additional studies.

\section{Inclusion and exclusion criteria}

Articles were included if they: reported on empirical evidence; were published in peer-reviewed journals; were written in English; and reported on actual or hypothetical disclosure. Psychological effects were defined as effects on emotions, mood and cognition. Behavioral effects were defined as changes in behavior that were likely to be caused by the disclosure. Social effects were defined as effects on individuals in their social context. Studies were excluded if they concerned participants who were previously diagnosed with dementia or MCI; or who had a family history of monogenetic AD. If multiple articles reported on the same outcome measures of a single study, the most relevant or extensive report was selected. Excluded studies and reasons for exclusion were documented (see Fig. 1).

\section{Study selection}

Duplicates were removed after retrieving records from the search strategy. In the screening phase, titles and abstracts were independently screened by three reviewers (SASAB, KT and $\mathrm{EMB}$ ) for eligibility to the research question. In the eligibility phase, full-text articles were assessed by the same three reviewers. In the case of discrepancy between the reviewers, consensus was reached after discussion.

\section{Data extraction and study quality assessment}

Data were extracted from the included studies by one reviewer (SASAB) using a data extraction form (see Additional file 2) that was collaboratively designed and piloted (KT, EMB and ER). We extracted data regarding the main outcome measures (psychological, behavioral and social effects), and additional information on study 


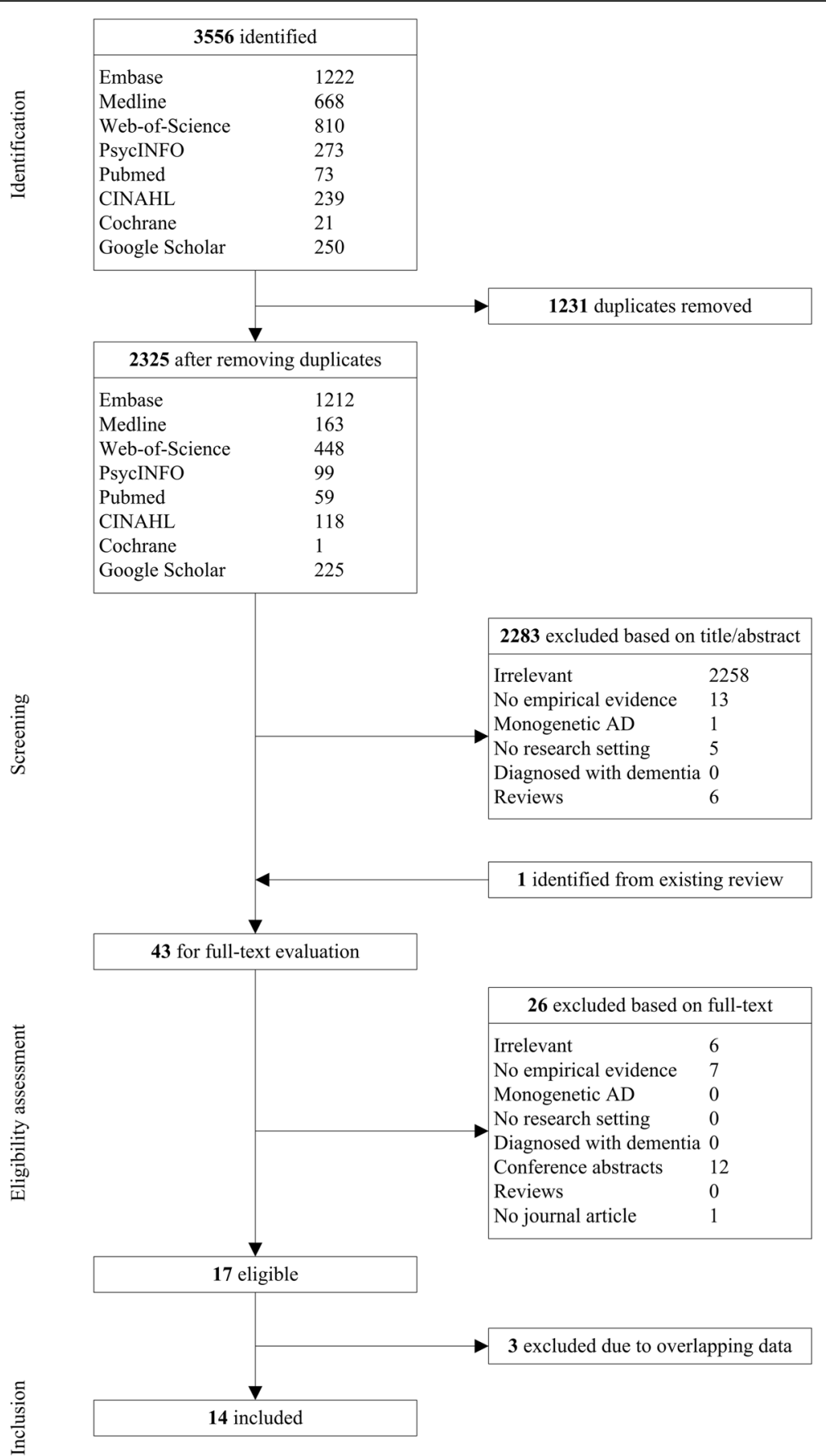

Fig. 1 PRISMA flow diagram illustrating process of study selection. In the last step, three studies were excluded due to overlapping data. Fanshawe et al. [53] was excluded because the behavioral outcomes relevant to our research question are also reported in Chao et al. [22]. Cassidy et al. [54] was excluded because the psychological effects relevant to our research question are described more extensively in Green et al. [23] Finally, Roberts et al. [55] was excluded because the psychological effects are described in Green et al. [23] and the behavioral effects in Chao et al. [22] and Zick et al. [24]. AD Alzheimer's disease, CINAHL Cumulative Index to Nursing and Allied Health Literature

design, characteristics and population, content and method of disclosure, other outcome measures and funding sources. The risk of bias in quantitative studies was evaluated with the Cochrane Collaboration's tool for assessing risk of bias, insofar as checklist items were applicable [20]. Qualitative studies were assessed with the Critical Appraisal Skills Programme checklist for quality assessment of qualitative research [21]. Studies with a high risk of bias were excluded from data synthesis. 


\section{Data synthesis}

Reported outcomes were both quantitative and qualitative in nature and varied widely between studies, so we composed a metasummary of the reported effects. First, we extracted data on the effects of disclosure from the results sections of the eligible studies. Second, we grouped these effects per outcome measure: psychological, behavioral or social effects.

\section{Results}

\section{Study selection and quality assessment}

A PRISMA flowchart for the results of the study selection is shown in Fig. 1. The electronic database searches generated 2325 unique records. After screening titles and abstracts, 43 records were evaluated in full text for eligibility. After this evaluation, 17 articles were deemed eligible. Three articles were excluded due to overlapping data, because they reported on the same outcome measures of a single study as other included articles. Fourteen articles were eventually identified for data extraction and data synthesis.

For each article, the main potential types and sources of bias were identified (see Additional file 3). All 14 articles assessed were considered not to have a high risk of bias and to be of sufficient quality for inclusion in the data synthesis of this systematic review [22-35].

\section{Study characteristics of included studies}

Of the 14 included studies, two were qualitative studies (interviews) and 12 were quantitative studies. The qualitative studies included 60 and 79 participants $[25,26]$. The populations of the quantitative studies varied between 76 and 343 participants, with the exception of one article describing two studies $(n=743)$ [27] and an online survey on hypothetical risk disclosure $(n=4036)$ [28]. In most studies, follow-up assessments were conducted after 6 weeks, 6 months and 12 months. All 14 studies were performed in the USA. Of these 14 studies, 11 reported on data collected in the REVEAL studies. The REVEAL studies are multicenter randomized controlled trials examining the effects of (REVEAL I) and different methods for (REVEAL II and III) APOE genotype disclosure in first-degree relatives (FDRs) of patients with AD. In the REVEAL I study, participants underwent APOE genetic testing and were randomized to receive either their genotype and a lifetime $\mathrm{AD}$ risk estimate incorporating this result (the disclosure group) or a risk estimate on the basis of family history, sex and age only (the nondisclosure group) [23]. In the REVEAL II study, everyone received their APOE genotype and participants were randomized to an extended or condensed disclosure protocol with either a face-to-face meeting or an information brochure prior to testing, while both groups had a face-to-face disclosure session [29]. No articles on the actual disclosure of nongenetic biomarkers were identified, although the only article describing hypothetical consequences of disclosure referred to nongenetic biomarker testing [28]. Whereas most studies examined the difference in outcome of interest between those receiving $\varepsilon 4$-positive and $\varepsilon 4$-negative results, none of them analyzed $\varepsilon 4$ heterozygotes and $\varepsilon 4$ homozygotes separately. Table 1 presents an overview of the study characteristics.

\section{Data synthesis}

Of the 14 included studies, six reported on psychological effects, six on behavioral effects and four on social effects (two studies reported on two types of effects). Results from all included studies are summarized in Table 2.

\section{Psychological effects}

Six studies reported on psychological effects, including anxiety, depression, test-related distress, coping and memory functioning [23, 25, 29-32]. In three studies, anxiety, depression and test-related distress levels were determined with the Beck Anxiety Inventory (BAI), the Center for Epidemiological Studies Depression Scale (CES-D) and the Impact of Event Scale (IES) respectively $[23,29,31]$. One study evaluated coping by means of a self-developed questionnaire [30], and another via interviews [25]. One study assessed objective memory functioning using the Logical memory subtest of the Wechsler Memory Scale Revised (WMS-R) and the Rey-Osterrieth Complex Figure Test (ROCFT), and assessed subjective memory functioning with the Metamemory in Adulthood (MIA) questionnaire and the Memory Functioning Questionnaire (MFQ) [32].

\section{Anxiety}

REVEAL I showed that, in FDRs of AD patients, there were no differences in anxiety levels between APOE $\varepsilon 4$-positive participants, $\varepsilon 4$-negative participants and the nondisclosure group [23, 29]. REVEAL II showed that anxiety levels were equal among the different disclosure protocols [29]. In both studies, anxiety levels on the BAI were on average below the clinical cutoff score of 16 [23,29]. Telling the testing result to a friend was associated with a decrease in anxiety level [31].

\section{Depression}

REVEAL I showed no differences in postdisclosure depression levels between $\varepsilon 4$ carriers, $\varepsilon 4$-negative participants and the nondisclosure group [23]. In REVEAL II, depression levels were equal in the different disclosure protocols [29]. In both studies, depression levels on the CES-D were on average below the clinical cutoff point of 16 [23, 29]. Twenty-four REVEAL II participants (9\%) reported depression scores above the clinical cutoff point 12 months post disclosure, and 21 of these 24 had 
Table 1 Characteristics of studies included in the systematic review

\begin{tabular}{|c|c|c|c|c|c|c|c|c|}
\hline Authors & Year & Country & $\begin{array}{l}\text { Study } \\
\text { name }\end{array}$ & $\begin{array}{l}\text { Study } \\
\text { design }\end{array}$ & $\begin{array}{l}\text { Study } \\
\text { population (n) }\end{array}$ & Disclosure & $\begin{array}{l}\text { Assessment } \\
\text { timepoints }\end{array}$ & Outcome measures \\
\hline \multicolumn{9}{|c|}{ Studies on psychological effects } \\
\hline Romero et al. [30] & 2005 & USA & & Cohort & 76 & $\begin{array}{l}\text { APOE } \\
\text { genotype }\end{array}$ & $\begin{array}{l}1 \text { month, } 4 \text { months, } \\
10 \text { months }\end{array}$ & Emotional reactions \\
\hline Gooding et al. [25] & 2006 & USA & REVEAL QRI & Qualitative & 60 & $\begin{array}{l}\text { APOE } \\
\text { genotype }\end{array}$ & $>1$ year & Coping \\
\hline Green et al. [23] & 2009 & USA & REVEAL I & RCT & 162 & $\begin{array}{l}\text { APOE } \\
\text { genotype }\end{array}$ & $\begin{array}{l}\text { Baseline, } 6 \text { weeks, } \\
6 \text { months, } 1 \text { year }\end{array}$ & $\begin{array}{l}\text { Anxiety, depression, test-related } \\
\text { distress }\end{array}$ \\
\hline Ashida et al. [31] & 2010 & USA & REVEAL ॥ & $\mathrm{RCT}$ & 269 & $\begin{array}{l}\text { APOE } \\
\text { genotype }\end{array}$ & $\begin{array}{l}\text { Baseline, } 6 \text { weeks, } \\
1 \text { year }\end{array}$ & $\begin{array}{l}\text { Relation results communication } \\
\text { and anxiety, depression and } \\
\text { test-related distress }\end{array}$ \\
\hline Lineweaver et al. [32] & 2014 & USA & & Case-control & 144 & $\begin{array}{l}\text { APOE } \\
\text { genotype }\end{array}$ & $\begin{array}{l}\text { Mean } 8.2 \text { months } \\
\text { post disclosure }\end{array}$ & $\begin{array}{l}\text { Objective and subjective memory } \\
\text { functioning }\end{array}$ \\
\hline Green et al. [29] & 2014 & USA & REVEAL ॥ & $\mathrm{RCT}$ & 343 & $\begin{array}{l}\text { APOE } \\
\text { genotype }\end{array}$ & $\begin{array}{l}\text { Baseline, } 6 \text { weeks, } \\
6 \text { months, } 1 \text { year }\end{array}$ & Anxiety, depression, distress \\
\hline \multicolumn{9}{|c|}{ Studies on behavioral effects } \\
\hline Zick et al. [24] & 2005 & USA & REVEAL I & $\mathrm{RCT}$ & 162 & $\begin{array}{l}\text { APOE } \\
\text { genotype }\end{array}$ & $\begin{array}{l}6 \text { weeks, } 6 \text { months, } \\
1 \text { year }\end{array}$ & Insurance uptake \\
\hline Chao et al. [22] & 2008 & USA & REVEAL I & RCT & 162 & $\begin{array}{l}\text { APOE } \\
\text { genotype }\end{array}$ & 1 year & $\begin{array}{l}\text { Health-related behavior: diet, } \\
\text { physical exercise, medication/ } \\
\text { vitamin intake }\end{array}$ \\
\hline Vernarelli et al. [33] & 2010 & USA & REVEAL ॥ & $\mathrm{RCT}$ & 272 & $\begin{array}{l}\text { APOE } \\
\text { genotype }\end{array}$ & 6 weeks & $\begin{array}{l}\text { Health-related behavior: dietary } \\
\text { supplement intake }\end{array}$ \\
\hline Taylor et al. [34] & 2010 & USA & REVEAL ॥ & RCT & 276 & $\begin{array}{l}\text { APOE } \\
\text { genotype }\end{array}$ & Not reported & Insurance uptake \\
\hline $\begin{array}{l}\text { Christensen } \\
\text { et al. [27] }\end{array}$ & 2015 & USA & REVEAL I/III & $\mathrm{RCT}$ & 795 & $\begin{array}{l}\text { APOE } \\
\text { genotype }\end{array}$ & 6 weeks, 1 year & $\begin{array}{l}\text { Insurance uptake, health-related } \\
\text { behavior and other behavior } \\
\text { changes }\end{array}$ \\
\hline $\begin{array}{l}\text { Hypothetical Caselli } \\
\text { et al. [28] }\end{array}$ & 2014 & USA & & Survey & 4036 & $\begin{array}{l}\text { Hypothetical } \\
\text { disclosure }\end{array}$ & Not applicable & $\begin{array}{l}\text { Anticipated health-related behavior } \\
\text { and other behavior changes }\end{array}$ \\
\hline \multicolumn{9}{|l|}{ Studies on social effects } \\
\hline Ashida et al. [35] & 2009 & USA & REVEAL ॥ & RCT & 271 & $\begin{array}{l}\text { APOE } \\
\text { genotype }\end{array}$ & 6 weeks & Results communication \\
\hline Ashida et al. [31] & 2010 & USA & REVEAL ॥ & RCT & 269 & $\begin{array}{l}\text { APOE } \\
\text { genotype }\end{array}$ & $\begin{array}{l}\text { Base, } 6 \text { weeks, } \\
1 \text { year }\end{array}$ & $\begin{array}{l}\text { Relation results communication } \\
\text { and anxiety, depression and } \\
\text { test-related distress }\end{array}$ \\
\hline Chilibeck et al. [26] & 2011 & Canada/USA & REVEAL I & Qualitative & 79 & $\begin{array}{l}\text { APOE } \\
\text { genotype }\end{array}$ & Not reported & $\begin{array}{l}\text { Risk perception in relation to } \\
\text { family history }\end{array}$ \\
\hline $\begin{array}{l}\text { Hypothetical Caselli } \\
\text { et al. [28] }\end{array}$ & 2014 & USA & & Survey & 4036 & $\begin{array}{l}\text { Hypothetical } \\
\text { disclosure }\end{array}$ & Not applicable & Anticipated results communication \\
\hline
\end{tabular}

Of the 14 studies included in the systematic review, two report on two types of effects and are taken up twice in this table. Ashida et al. [31] report on both psychological and social effects, and Caselli et al. [28] report on both behavioral and social effects

$A P O E$ apolipoprotein E, RCT randomized controlled trial, REVEAL Risk Evaluation and Education for Alzheimer's Disease Study

scores below this cutoff score at baseline [29]. Telling the testing result to a healthcare professional was associated with a decrease in depression level [31].

\section{Test-related distress}

REVEAL I showed that 6 weeks and 6 months, but not 12 months, after disclosure, $\varepsilon 4$-positive participants had higher test related distress levels than $\varepsilon 4$-negative participants. At 6 months, but not 6 weeks and 12 months, after disclosure, the nondisclosure group had higher test-related distress than $\varepsilon 4$-negative participants [23]. In REVEAL II, 6 weeks and 6 months after disclosure, test-related distress levels were higher in the condensed protocol group counseled by a medical doctor compared with the extended protocol group counseled by a genetic counselor. There were no differences in distress between the extended and condensed protocol groups that were both counseled by a genetic counselor [29]. Postdisclosure distress levels were on average below the threshold of 20, indicative of significant distress, in each group [23, 29]. No association was found between results communication and test-related distress levels [31].

\section{Coping}

One study measured emotional reactions to disclosure 1, 4 and 10 months post disclosure via a self-developed 
Table 2 Results of 14 included studies

\begin{tabular}{|c|c|c|c|c|c|c|c|c|c|}
\hline Authors, year & Research question/design & Sample $(n)$ & Outcome measures & Instruments & Time points & \multicolumn{4}{|l|}{ Results } \\
\hline \multicolumn{10}{|c|}{ Studies on psychological effects } \\
\hline \multirow{10}{*}{$\begin{array}{l}\text { Romero et al., } \\
2005 \text { [30] }\end{array}$} & \multirow{10}{*}{$\begin{array}{l}\text { Participants were asked how they felt } \\
\text { after disclosure of their APOE genotype }\end{array}$} & \multirow[t]{10}{*}{76} & \multirow{4}{*}{ Feeling depressed } & \multirow{10}{*}{$\begin{array}{l}\text { Self-developed } \\
\text { questionnaire }\end{array}$} & & \multicolumn{2}{|l|}{ ع4-negative group } & \multicolumn{2}{|c|}{ ع4-positive group } \\
\hline & & & & & 1 month & \multicolumn{2}{|l|}{$0 / 49$} & \multicolumn{2}{|l|}{$8 / 27$} \\
\hline & & & & & 4 months & \multicolumn{2}{|l|}{$0 / 49$} & \multicolumn{2}{|l|}{$5 / 27$} \\
\hline & & & & & 10 months & \multicolumn{2}{|l|}{$0 / 47$} & \multicolumn{2}{|l|}{$4 / 27$} \\
\hline & & & \multirow[t]{3}{*}{ Feeling worried } & & 1 month & \multicolumn{2}{|l|}{$0 / 49$} & \multicolumn{2}{|l|}{$6 / 27$} \\
\hline & & & & & 4 months & \multicolumn{2}{|l|}{$0 / 49$} & \multicolumn{2}{|l|}{$3 / 27$} \\
\hline & & & & & 10 months & \multicolumn{2}{|l|}{$0 / 47$} & \multicolumn{2}{|l|}{$3 / 27$} \\
\hline & & & \multirow[t]{3}{*}{ Feeling relieved } & & 1 month & \multicolumn{2}{|l|}{$36 / 49$} & \multicolumn{2}{|l|}{$4 / 27$} \\
\hline & & & & & 4 months & \multicolumn{2}{|l|}{$30 / 49$} & \multicolumn{2}{|l|}{$5 / 27$} \\
\hline & & & & & 10 months & \multicolumn{2}{|l|}{$34 / 47$} & \multicolumn{2}{|l|}{$3 / 27$} \\
\hline \multirow{4}{*}{$\begin{array}{l}\text { Gooding et al., } \\
\text { 2006 (REVEALL } \\
\text { QRI) [25] }\end{array}$} & \multirow{4}{*}{$\begin{array}{l}56 \text { participants in the REVEAL I study and } \\
\text { four individuals who declined participation } \\
\text { were interviewed about their reaction to } \\
\text { the received results (either risk estimate } \\
\text { and genotype or risk estimate only) }\end{array}$} & \multirow{4}{*}{$60^{\mathrm{a}}$} & Coping: & \multirow[t]{4}{*}{ Interviews } & $>12$ months & \multicolumn{2}{|l|}{ ع4-positive group } & $\begin{array}{l}\text { Other } \\
\text { REVEA }\end{array}$ & D, not \\
\hline & & & Relief & & & $\begin{array}{l}4 / 17(24 \%) \text { were } \\
\text { "Even with the } \varepsilon 4 \\
\text { their risk was not a } \\
\text { as they had previo } \\
\text { anticipated" }\end{array}$ & $\begin{array}{l}\text { relieved: } \\
\text { allele, } \\
\text { as high } \\
\text { pusly }\end{array}$ & $25 / 43$ & ieved $^{\mathrm{b}}$ \\
\hline & & & Worry & & & $\begin{array}{l}\text { Six participants, all } \\
\text { expressed greater } \\
\text { concern about the } \\
\text { risk after disclosure } \\
\text { describing their res } \\
\text { "depressing", "frigh } \\
\text { and "disappointing }\end{array}$ & $\begin{array}{l}\text { \&4+, } \\
\text { eir AD } \\
\text { e, } \\
\text { sults as } \\
\text { tening" } \\
\text { g" }\end{array}$ & $\begin{array}{l}14 / 39 \\
\text { REVEA } \\
\text { relieve }\end{array}$ & $\begin{array}{l}\text { larticipated in } \\
\text { eneither } \\
\text { worried }\end{array}$ \\
\hline & & & Neither & & & $\begin{array}{l}7 / 17 \text { were neither } \\
\text { relieved nor worrie } \\
\text { They related their } \\
\text { emotion to the lac } \\
\text { predictability of th } \\
\text { test and the feelin } \\
\text { it only confirmed } \\
\text { they had already } \\
\text { accept as their risk }\end{array}$ & $\begin{array}{l}\text { ed. } \\
\text { "lack of } \\
\text { ck of } \\
\text { le APOE } \\
\text { ig that } \\
\text { what } \\
\text { come to } \\
\text { < for AD" }\end{array}$ & & \\
\hline $\begin{array}{l}\text { Green et al., } \\
2009 \text { [23] }\end{array}$ & $\begin{array}{l}\text { Participants were randomly assigned to } \\
\text { receive their APOE genotype and a risk }\end{array}$ & 162 & & & & ع4-positive group & $\varepsilon 4$-negati & group & $\begin{array}{l}\text { No disclosure } \\
\text { group }\end{array}$ \\
\hline (REVEAL I) & $\begin{array}{l}\text { estimate (ह4-positive and } \varepsilon 4 \text {-negative } \\
\text { group) or a risk estimate only (no }\end{array}$ & & Anxiety & BAI (0-63) & 6 weeks & $5.2(0.7)^{c}$ & $4.5(0.6)$ & & $4.4(0.7)^{c}$ \\
\hline & disclosure group) & & & & 6 months & $4.6(0.6)$ & $3.9(0.6)$ & & $4.6(0.6)$ \\
\hline & & & & & 12 months & $4.4(0.6)$ & $4.2(0.6)$ & & $4.2(0.6)$ \\
\hline
\end{tabular}


Table 2 Results of 14 included studies (Continued)

\begin{tabular}{|c|c|c|c|c|c|c|c|c|c|}
\hline & & & Depression & CES-D (0-60) & 6 weeks & $9.0(1.0)$ & \multicolumn{2}{|l|}{$8.5(0.9)$} & $9.3(1.0)$ \\
\hline & & & & & 6 months & $9.6(1.0)$ & \multicolumn{2}{|l|}{$8.9(1.0)$} & $8.7(1.0)$ \\
\hline & & & & & 12 months & $8.3(0.9)$ & \multicolumn{2}{|l|}{$8.5(0.9)$} & $8.0(0.9)$ \\
\hline & & & \multirow[t]{3}{*}{ Distress } & IES (0-75) & 6 weeks & $9.4(1.3)^{d}$ & \multicolumn{2}{|l|}{$5.2(1.3)$} & $6.7(1.4)$ \\
\hline & & & & & 6 months & $8.6(1.2)^{d}$ & \multicolumn{2}{|l|}{$4.2(1.2)$} & $8.9(1.3)^{\mathrm{e}}$ \\
\hline & & & & & 12 months & $8.5(1.3)$ & \multicolumn{2}{|l|}{$5.1(1.2)$} & $7.7(1.5)$ \\
\hline \multirow[t]{5}{*}{$\begin{array}{l}\text { Ashida et al., } \\
2010 \text { (REVEAL II) } \\
\text { [31] }\end{array}$} & \multirow[t]{5}{*}{$\begin{array}{l}\text { Participants in the REVEAL II study all } \\
\text { received their APOE genotype, and were } \\
\text { randomly assigned to either an extended } \\
\text { or condensed disclosure protocol }\end{array}$} & \multirow[t]{5}{*}{269} & $\begin{array}{l}\text { Effect of results } \\
\text { communication on } \\
\text { psychological well-being: }\end{array}$ & $\begin{array}{l}\text { "Have you told } \\
\text { anyone your APOE } \\
\text { genetic test result? } \\
\text { If so, who?" }\end{array}$ & 6 weeks & \multicolumn{4}{|c|}{$\begin{array}{l}62.1 \% \text { told a family member, } 52 \% \text { their spouse or } \\
\text { significant other, } 37.5 \% \text { a friend and } 14.9 \% \text { a health } \\
\text { professional }\end{array}$} \\
\hline & & & Anxiety & $\mathrm{BAl}$ & 12 months & \multicolumn{4}{|c|}{$\begin{array}{l}\text { Telling the result to a friend was associated with a } \\
\text { decrease in BAl at } 12 \text { months (regression coefficient } \\
b=-0.17, p<0.01 \text { ) }\end{array}$} \\
\hline & & & Depression & CES-D & & \multicolumn{4}{|c|}{$\begin{array}{l}\text { Telling the result to a healthcare professional with a } \\
\text { decrease in CES-D at } 12 \text { months (regression coefficient } \\
b=-0.10, p<0.05 \text { ) }\end{array}$} \\
\hline & & & \multirow[t]{2}{*}{ Distress } & \multirow[t]{2}{*}{ IGT-AD $(0-60)$} & & \multirow{2}{*}{\multicolumn{4}{|c|}{$\begin{array}{l}\text { There was no association between results communication } \\
\text { and IGT-AD levels of distress } \\
24 \text { participants ( } 9 \% \text { ) reported scores > than the clinical } \\
\text { cutoff point of } 16 \text { on the CES-D at } 12 \text { months, and } 21 \\
\text { of these } 24 \text { had scores < the cutoff at baseline }\end{array}$}} \\
\hline & & & & & & & & & \\
\hline \multirow[t]{9}{*}{$\begin{array}{l}\text { Lineweaver } \\
\text { et al., } 2014 \text { [32] }\end{array}$} & \multirow{9}{*}{$\begin{array}{l}\text { A group of research participants who } \\
\text { were informed about their APOE } \\
\text { genotype was compared with a matched } \\
\text { group who did not receive their genotype } \\
\text { on objective and subjective memory } \\
\text { functioning }\end{array}$} & \multirow[t]{9}{*}{144} & \multirow{9}{*}{$\begin{array}{l}\text { Objective memory } \\
\text { functioning Subjective } \\
\text { memory functioning }\end{array}$} & $\begin{array}{l}\text { Logical memory } \\
\text { subtest }\end{array}$ & \multirow[t]{9}{*}{$\begin{array}{l}\text { Mean } \\
8.2 \text { months }\end{array}$} & $\begin{array}{l}\text { ع4-positive } \\
\text { uninformed }\end{array}$ & $\begin{array}{l}\varepsilon 4 \text {-positive } \\
\text { informed }\end{array}$ & $\begin{array}{l}\text { घ4-negative } \\
\text { uninformed }\end{array}$ & $\begin{array}{l}\varepsilon 4 \text {-negative } \\
\text { informed }\end{array}$ \\
\hline & & & & immediate recall & & $31.9(8.5)^{\mathrm{h}}$ & $22.9(7.8)$ & $28.4(7.8)$ & $25.6(5.9)$ \\
\hline & & & & $\begin{array}{l}\text { delayed recall } \\
\text { Wechsler Memory } \\
\text { Scale Revised (0-50) }\end{array}$ & & $28.1(9.3)^{\mathrm{h}}$ & $17.7(7.9)$ & $24.3(9.6)$ & $20.6(6.9)$ \\
\hline & & & & \multirow{2}{*}{$\begin{array}{l}\text { Immediate recall } \\
\text { delayed recall on } \\
\text { Rey-Osterrieth } \\
\text { Complex Figure } \\
\text { test }(0-20)\end{array}$} & & $9.7(2.7)$ & $10.6(2.6)$ & $9.3(3.7)$ & $9.7(3.1)$ \\
\hline & & & & & & $9.0(3.1)$ & $10.1(2.4)$ & $9.0(4.0)$ & $9.9(2.8)$ \\
\hline & & & & $\begin{array}{l}\text { Metamemory in } \\
\text { Adulthood } \\
\text { Questionnaire: }\end{array}$ & & & & & \\
\hline & & & & \multirow{2}{*}{$\begin{array}{l}\text { Capacity subscale } \\
\text { Change over time } \\
\text { subscale (15 items } \\
\text { 5-point Likert scale) }\end{array}$} & & $31.2(5.2)^{i}$ & $28.0(6.4)$ & $27.8(6.5)^{j}$ & $30.5(4.7)$ \\
\hline & & & & & & $17.0(5.4)$ & $16.9(5.5)$ & $15.9(4.9)$ & $16.7(4.1)$ \\
\hline & & & & $\begin{array}{l}\text { Memory Functioning } \\
\text { Questionnaire: }\end{array}$ & & & & & \\
\hline
\end{tabular}


Table 2 Results of 14 included studies (Continued)

Green et al., Participants were randomly assigned to 2014 (REVEAL II) receive their APOE genotype in an

extended protocol (SP-GC), a condensed

protocol with a genetic counselor (CP-GC)

or medical doctor (CP-MD). Aim was to

assess whether the condensed protocols

were equal $(=$ mean score on any of the

scales not more than 5 points higher) to the extended protoco

Studies on behavioral effects

Zick et al., 2005 REVEAL I, see Green et al., 2009 [23] (REVEAL I) [24]

Anxiety
Depression
Distress

$\begin{array}{ll} & \text { Retrospective } \\ \text { functioning } & \\ & \text { Frequency of } \\ & \text { forgetting } \\ & \text { Forgetting when } \\ & \text { reading } \\ & \text { Forgetting past } \\ & \text { events } \\ & \text { Mnemonics usage } \\ & \text { (46 items with } \\ \text { 7-point Likert scale) }\end{array}$

BAI (0-63)

CES-D (0-60)

162
Insurance uptake
Questionnaires on
$18.3(5.8)$

90.5 (14.7)

$15.9(4.7)$

$14.3(4.1)^{k}$

$16.7(4.7)$

$54.9(8.4)$

$83.4(13.3) \quad 84.5(17.0) \quad 88.9(13.4)$

$(19.6)$

$50.7(9.3)$

$50.5(13.1)^{\mathrm{k}} \quad 55.5(8.8)$

$19.2(4.6)$

18.3 (4.3)

$18.1(4.9)$

$19.9(4.5)$

$23.1(11.4)$

$20.3(7.4)$

$22.8(9.5)$

$25.1(8.4)$

SP-GC

CP-GC

CP-MD

$2.6(0.5)^{c}$

$3.6(0.5)$

$3.1(0.5)$

$4.3(0.5)$

6 months $3.2(0.5)$

$3.7(0.5)$

$5.8(0.7)$

$5.8(0.7)$

6 weeks $\quad 5.7(0.7)$

$5.8(0.7)$
$5.6(0.6)$

$5.1(0.9)$

$4.0(0.9)$

$3.3(0.8)$

$4.4(0.5)$

$3.9(0.5)$

$8.1(0.7)$

$8.1(0.7)$

$6.9(0.6)$

$8.2(0.9)^{\prime}$

$7.0(0.9)^{1}$

$5.5(0.8)$ actual change in

6 weeks,

6 months,

12 months

Health insurance

Life insurance

Disability insurance

LTC insurance (\%)

Planned change in

Health insurance

Life insurance

Disability insurance

LTC insurance (\%) $\varepsilon 4$-positive group $\varepsilon 4$-negative group No disclosure group

6.52

6.52

4.35

4.35

23.9

4.35

8.70

32.6 


\begin{tabular}{|c|c|c|c|c|c|c|c|c|c|}
\hline \multirow{3}{*}{$\begin{array}{l}\text { Taylor et al., } \\
2010 \text { (REVEAL II) } \\
\text { [34] }\end{array}$} & \multirow[t]{3}{*}{ REVEAL II, see Green et al., 2014 [29] } & \multirow[t]{3}{*}{276} & LTC insurance & \multirow[t]{3}{*}{ Not reported } & \multirow[t]{3}{*}{$\begin{array}{l}\text { Not } \\
\text { reported }\end{array}$} & Two $\varepsilon 3$ traits & \multicolumn{2}{|c|}{$\geq$ one $\varepsilon 4$ trait } & $\begin{array}{l}\text { At least one } \varepsilon 2 \\
\text { trait, no } \varepsilon 4 \text { trait }\end{array}$ \\
\hline & & & $\begin{array}{l}\text { OR of actual or planned } \\
\text { change }\end{array}$ & & & 1.00 & \multicolumn{2}{|c|}{$2.31(95 \% \mathrm{Cl} 1.11-4.81)$} & $\begin{array}{l}1.55(95 \% \mathrm{Cl} \\
0.43-5.60)\end{array}$ \\
\hline & & & $\begin{array}{l}\text { Absolute probability of } \\
\text { changing LTC insurance }\end{array}$ & & & 0.087 & \multicolumn{2}{|l|}{0.237} & 0.149 \\
\hline \multirow{6}{*}{$\begin{array}{l}\text { Chao et al., } \\
2008 \text { (REVEAL I) } \\
\text { [22] }\end{array}$} & \multirow[t]{6}{*}{ REVEAL I, see Green et al., 2009 [23] } & \multirow[t]{6}{*}{162} & \multirow[t]{6}{*}{$\begin{array}{l}\text { Health-related behavior } \\
\text { changes }\end{array}$} & $\begin{array}{l}\text { Yes/no questions } \\
\text { about changes in: }\end{array}$ & \multirow[t]{6}{*}{12 months } & ع4-positive group & \multicolumn{2}{|c|}{$\varepsilon 4$-negative group } & $\begin{array}{l}\text { no disclosure } \\
\text { group }\end{array}$ \\
\hline & & & & $\begin{array}{l}\text { Any behavior } \\
\text { specific to } A D \\
\text { prevention }\end{array}$ & & $52^{n}$ & \multicolumn{2}{|l|}{24} & 30 \\
\hline & & & & Medications/vitamins & & 40 & \multicolumn{2}{|l|}{20} & 28 \\
\hline & & & & Diet & & 20 & \multicolumn{2}{|l|}{11} & 7 \\
\hline & & & & \multirow{2}{*}{$\begin{array}{l}\text { Exercise } \\
\text { (\% endorsing) }\end{array}$} & & 8 & \multicolumn{2}{|l|}{4} & 5 \\
\hline & & & & & & $\begin{array}{l}\text { OR of any } \\
\text { behavior change } \\
\text { ع4+ vs } \varepsilon 4- \\
\text { group: } 2.73 \\
(95 \% \mathrm{Cl} \\
1.14-6.54)\end{array}$ & & & \\
\hline \multirow{5}{*}{$\begin{array}{l}\text { Vernarelli et al., } \\
2010 \text { (REVEAL II) } \\
\text { [33] }\end{array}$} & \multirow[t]{5}{*}{ REVEAL II, see Green et al., 2014 [29] } & \multirow[t]{5}{*}{272} & \multirow{5}{*}{$\begin{array}{l}\text { Changes in supplement } \\
\text { use }\end{array}$} & \multirow{5}{*}{$\begin{array}{l}\text { Yes/no questions } \\
\text { with free-text field } \\
\text { on changes in: } \\
\text { overall diet use of } \\
\text { dietary supplements } \\
\text { exercise (ORs) }\end{array}$} & \multirow[t]{5}{*}{6 weeks } & \multicolumn{2}{|l|}{ ع4-positive group } & \multicolumn{2}{|c|}{$\varepsilon 4$-negative group } \\
\hline & & & & & & \multicolumn{2}{|c|}{$1.56(95 \%$ Cl 0.80-3.02) } & \multicolumn{2}{|l|}{1.00} \\
\hline & & & & & & \multicolumn{2}{|c|}{$\begin{array}{l}4.75(p<0.0001) \\
(95 \% \mathrm{Cl} 2.23-10.10)\end{array}$} & \multicolumn{2}{|l|}{1.00} \\
\hline & & & & & & \multicolumn{2}{|c|}{$1.85(95 \% \mathrm{Cl} 0.96-3.57)$} & 1.00 & \\
\hline & & & & & & \multicolumn{4}{|c|}{$\begin{array}{l}\text { Of } 45 \text { participants reporting } \\
\text { a change in supplement use, } \\
32(71.1 \%) \text { were } \varepsilon 4+ \\
(p<0.0001) \text {. Of these } 45,38 \\
\text { were in the condensed } \\
\text { protocol }(84.4 \%) \text { and seven } \\
\text { in the extended }(15.5 \%) \\
(p=0.006)\end{array}$} \\
\hline
\end{tabular}

OR for actual change in LTC insurance $\varepsilon 4+$ compared with ND: 5.76 $(p<0.1)$

Taylor et al., REVEAL II, see Green et al, 2014 [29] 276 2010 (REVEAL II)

[34]

OR of actual or planned change

REVEAL I, see Green et al., 2009 [23]

Health-related behavior

Medications/vitamins

Yes/no questions dietary supplements 
Table 2 Results of 14 included studies (Continued)

Christensen Secondany analyses were performed on et al., 2015 data from the REVEAL II and III study. For

(REVEAL II/III) REVEAL II, see Green et al., 2014 [29]. In

[27]

the REVEAL III study, in-person and phon
disclosure, and giving AD genetic info

only and pleiotropic info were compared

Associations between recruitment status

(actively recruited (ARP)

or self-referred (SRP)) and

or self-referred (SRP)) and

advance planning

Health behavior:

activities Diet

Exercise Dietary

supplements

Advance planning:

LTC insurance

Retirement plans

Studies on hypothetical disclosure

Caselli et al., Members of an online community for

2014 [28] people interested in AD prevention

research completed a survey on their

interest in and anticipated reaction to

hypothetical genetic and biomarker

testing and disclosure
4036
Results communication
Multiple-choice
questions

Not

applicable
Self-referred participants were more likely than ARPs to report changes to exercise at 12 months (35 $p=0.032$ ). No other differences between recruitment cohorts were noted on changes or plans to change health behaviors

Secondary analyses showed that the impact of genetic risk status on certain behavior changes differed by recruitment cohort. $\varepsilon 4$-positive participants were more likely than $\varepsilon 4$-negative participants to report changes at 6 weeks to mental activities and diet, but only if they had self-referred to the study, although only differences in changes to

mental activities persisted through the 12-month follow-up

No direct associations with self-referral were observed on either LTC insurance coverage or retirement plans. An interaction effect was observed $(p=0.005)$ : self-referred $\varepsilon 4$-positive participants were more likely than $\varepsilon 4$-negative participants to report intentions to change LTC coverage, but no differences were noted among ARPs

No associations were noted on retirement plans, except greater intentions to change among $\varepsilon 4$-positive participants compared with $\varepsilon 4$-negative participants, regardless of recruitment cohort $(p<0.001)$

If APOE $\varepsilon 4$ positive, you

would tell:

$\begin{array}{ll}\text { Physician } & 79.4 \\ \text { Spouse } & 92.3 \\ \text { Siblings } & 84.6 \\ \text { Children } & 81.7 \\ \text { Friends } & 53 \\ \text { Lawyer } & 60\end{array}$

If biomarker evidence of $\mathrm{AD}$,

you would tell:

Spouse

Sibling

80.6

Children

Friends

Lawyer 
Table 2 Results of 14 included studies (Continued)

Behavior changes

\section{Studies on social effects}

Ashida et al., REVEAL II, see Green et al., 2014 [29] 2009 (REVEAL II)

Chilibeck et al., Interviews were conducted with

2011 (REVEAL I) participants from REVEAL I and with

[26]

FDRs of AD patients who did not

undergo genetic testing
Have you told anyone your APOE genetic test result?

If so, who?"

$\begin{array}{lll}79 \text { (REVEAL } & \text { Effect of personalized } & \text { Open-ended } \\ \text { participants) } & \text { genetic information on } & \text { interviews } \\ \text { and } 40 & \text { conceptualization of } & \\ \text { (non-REVEAL) } & \begin{array}{l}\text { personal risk, family } \\ \text { health and familial } \\ \\ \\ \text { relationships }\end{array}\end{array}$

If APOE $\varepsilon 4$ positive, you

\section{would:}

Begin a healthier lifestyle

Get LTC insurance

Spend all your money

forpleasure

Seriously consider suicide

If biomarker evidence of $A D$,

you would:

Begin a healthier lifestyle

Get LTC insurance

Spend all your money for

pleasure

Seriously consider suicide

6 weeks

Person

Anyone

Family member

Frequency (\%)

Spouse/significant other $\quad 50.9$

Friends

Health professional

OR of results communication

to health professional in

condensed vs extended

protocol $5.19(95 \% \mathrm{Cl}$

$1.50-17.89, p<0.01)$ reported
Drawn from the report: when genetic information corresponds with previous beliefs about risk and inheritance, people emphasize how the information provided by genetic testing is "not new" to them bu only confirms what they already knew or at least suspected. Yet risk predictions generated by genetic technologies sometimes conflict with those rooted in everyday beliefs about heredity. The visible evidence of risk provided by family history is often more compelling than that based on a genetic test 
Of the 14 studies included in the systematic review, two report on two types of effects. In this table they are taken up only once. Ashida et al. [31] report on both psychological and social effects; results from this study are to be found among the studies on psychological effects in this table. Caselli et al. [28] report on both behavioral and social effects; results from this study are to be found among the studies on behavioral effects in this table

AD Alzheimer's disease, APOE apolipoprotein E, ARP actively recruited participants, BAI Beck Anxiety Inventory, CES-D Center for Epidemiological Studies Depression Scale, CI confidence interval, CP-GC condensed protocol genetic counselor, CP-MD condensed protocol medical doctor, FDR first-degree relative, I informed, IES Impact of Event Scale, IGT-AD Impact of Genetic Testing for Alzheimer's Disease, LTC long-term care, ND no-disclosure group, OR odds ratio, RCT randomized controlled trial, REVEAL Risk Evaluation and Education for Alzheimer's Disease Study, SP-GC standard protocol genetic counselor, SRP self-referred participants, Ul uninformed

aAlthough the question of one's response to the results is not applicable to those who declined participation in REVEAL $(n=4)$, the original article does not leave those individuals out of this part of the results section ${ }^{b}$ Fifteen participants were in the no-disclosure group in REVEAL and received a lifetime risk estimate only. This group is not analyzed separately in their reaction to the results

'Scores are mean values $\pm \mathrm{SE}$

${ }^{\mathrm{d} D i f f e r e n c e} \varepsilon 4+$ vs $\varepsilon 4-$ is significant at 6 weeks and 6 months $(p<0.05)$

eDifference ND vs $\varepsilon 4$ - is significant at 6 months $(p<0.05)$

IIGT-AD based on the Multidimensional Impact of Cancer Risk Assessment Questionnaire

gOn all memory scales, higher scores indicate better objective/subjective memory functioning. Scores are mean values $\pm S D$

${ }^{\mathrm{h}} p \leq 0.001 \varepsilon 4+\mathrm{UI}$ vs $\varepsilon 4+1$

i $p<0.05 \varepsilon 4+\mathrm{Ul}$ vs $\varepsilon 4+$

${ }^{\mathrm{i}} p<0.05 \varepsilon 4-U \mathrm{Ul}$ vs $\varepsilon 4-1$

${ }^{k} p<0.05 \varepsilon 4-U$ U vs $\varepsilon 4-1$

'Noninferiority of CP-MD vs SP-GC could not be confirmed for test-related distress at 6 weeks and 6 months

$\mathrm{m}_{p}<0.05 \varepsilon 4+\mathrm{vs} \varepsilon 4$

${ }^{n} \varepsilon 4+$ vs $\varepsilon 4-, p=0.003 ; \varepsilon 4+$ vs ND, $p=0.03$ 
questionnaire: 61-73\% of ع4-negative participants and 11-19\% of ع4-positive participants felt relieved after disclosure. Of the \&4-positive participants, $15-30 \%$ felt depressed and 11-22\% felt worried after disclosure, compared with no individuals in the $\varepsilon 4$-negative group [30]. Of the REVEAL participants interviewed in the REVEAL Qualitative Research Initiative, $24 \%$ of $\varepsilon 4$-positive participants were relieved after receiving results, versus $58 \%$ of a group consisting of both $\varepsilon 4$-negative participants and individuals from the nondisclosure group. Thirty-five percent of $\varepsilon 4$-positive participants expressed greater concern about their risk for $\mathrm{AD}$, describing their results as "depressing", "frightening" and "disappointing", compared with no one in the other group. Forty-one percent of $\varepsilon 4$-positive participants were neither relieved nor worried. In the group consisting of $\varepsilon 4$-negative participants and people from the nondisclosure group, $36 \%$ of 39 participants felt neither relieved nor worried after learning their results [25].

\section{Memory functioning}

One study examined the effects of disclosure on objective and subjective memory functioning. Participants who were aware of being $\varepsilon 4$-negative rated multiple aspects of their own memory functioning higher on the MIA and the MFQ than $\varepsilon 4$-negative participants who did not know their genotype. Participants who knew they were $\varepsilon 4$-positive rated their memory functioning on the capacity subscale of the MIA lower, and performed worse on a logical memory subtest of the WMS-R than $\varepsilon 4$-positive participants who were not informed [32].

\section{Behavioral effects}

Six studies assessed one or more behavioral effects, including changes in insurance uptake, health-related behavior and other behavior [22, 24, 27, 28, 33, 34]. Five of those were part of the REVEAL studies [22, 24, 27, 33, 34]. Three studies examined actual and planned changes in insurance uptake within 1 year after disclosure via self-report questionnaires [24, 27, 34]. Health-related behavior changes were assessed by means of yes/no questions on specific behavior changes in three studies [22, 27, 33], and by asking "If APOE ع4-positive/biomarker evidence of $\mathrm{AD}$, you would" followed by several options in a study on hypothetical disclosure [28]. Two studies measured other behavioral effects, one via yes/no questions on specific actual or planned behavior changes [27] and one via the aforementioned question on hypothetical disclosure [28].

\section{Insurance uptake}

In REVEAL I, in the first year following disclosure, $16.7 \%$ of ع4-positive participants changed long-term care (LTC) insurance, versus $1.9 \%$ of $\varepsilon 4$-negative participants and $4.4 \%$ of the nondisclosure group. In addition, $45.8 \%$ of $\varepsilon 4$-positive participants planned to change LTC insurance, versus $22.2 \%$ of $\varepsilon 4$-negative participants and $32.6 \%$ of the nondisclosure group. No differences were found in changes to health, life or disability insurance uptake [24]. Data from REVEAL II show that $\varepsilon 4$-positive participants are 2.3 times more likely to report an actual or planned change to LTC insurance than people with two $\varepsilon 3$ alleles [34]. Secondary analyses of REVEAL II data indicate that the intention to change LTC coverage among $\varepsilon 4$-positive participants is limited to those who had self-referred to the study [27]. In a study of the hypothetical reaction to disclosure, $76 \%$ would get LTC insurance if APOE \&4-positive and $77 \%$ in the case of (nongenetic) biomarker evidence of $\mathrm{AD}$ [28].

\section{Health behavior}

REVEAL I showed that 12 months after disclosure, $\varepsilon 4$-positive participants reported changes in any one of the domains of diet, physical exercise and medication or vitamin intake, more often (52\%) than $\varepsilon 4$-negative participants (24\%) or the nondisclosure group (30\%). Within each domain, there were no significant differences between the groups [22]. In REVEAL II, 6 weeks after disclosure, ع4-positive participants were almost five times more likely to report changes to dietary supplement intake, but not to diet or physical exercise, than $\varepsilon 4$-negative participants [33]. Secondary analyses of REVEAL II pointed out that $\varepsilon 4$-positive participants were more likely to have changed diet 6 weeks after disclosure, mental activities 6 weeks after disclosure and 12 months after disclosure and medication intake 12 months after disclosure than \&4-negative participants, but only if they had self-referred to the study [27]. In a hypothetical disclosure scenario, $90.5 \%$ of respondents would adopt a healthier life style if APOE $\varepsilon 4$-positive and $91 \%$ in the case of having (nongenetic) biomarker evidence of $\mathrm{AD}[28]$.

\section{Other behavioral effects}

Two studies reported on other, planned, behavioral effects $[27,28]$. The REVEAL II study found greater intentions to change retirement plans among ع4-positive participants than 84-negative participants [27]. In the study on hypothetical disclosure, if people would be found to be \&4-positive or to have nongenetic biomarker evidence of $\mathrm{AD}, 18.4$ and $18.7 \%$ of respondents would spend all their money for pleasure and 11.6 and $10.2 \%$ agreed to the statement that they would seriously consider suicide [28].

\section{Social effects}

Four studies reported on social effects, namely the communication of results and the perception of risk in relation to family history $[26,28,31,35]$. Three studies examined results communication; two by asking participants in written questionnaires "Have you told anyone 
your result? If so, who?" [31, 35]; and one by asking hypothetically "If APOE \&4-positive/biomarker evidence of AD, you would tell ..." followed by several options [28]. The results of one of these studies, on the effects communication has on anxiety, depression and testrelated distress, are described in the psychological effects section [31]. Risk perception in relation to family history was studied in open-ended interviews [26].

\section{Results communication}

In REVEAL II, $81.5 \%$ of participants told at least one person their result; $63.8 \%$ told a family member, $50.9 \%$ their spouse or significant other, $34.7 \%$ a friend and $12.2 \%$ a health professional [35]. In a hypothetical scenario of being APOE ع4-positive, 79.4\% of respondents would tell their physician, $92.3 \%$ their spouse, $84.6 \%$ a sibling, $81.7 \%$ their children, $53 \%$ friends and $60 \%$ their lawyer. In case of having (nongenetic) biomarker evidence of $\mathrm{AD}, 92.2 \%$ would tell their spouse, $80.6 \%$ a sibling, $75.9 \%$ their children, $46.5 \%$ friends and $53.8 \%$ their lawyer [28].

\section{Perception}

In one study, interviews were conducted with two groups of FDRs of $\mathrm{AD}$ patients; the first group consisted of individuals who had undergone genetic testing (REVEAL participants), and the second contained people who had not received information on the genetics of $\mathrm{AD}$ [26]. Ideas on the causation of $\mathrm{AD}$ and personal risk of developing $\mathrm{AD}$ were found to be similar in these groups, suggesting that receiving information on the genetics of $\mathrm{AD}$ and undergoing genetic testing for $\mathrm{AD}$ does not strongly alter these ideas. The REVEAL participants included in this study considered genetic test results that corresponded with prior beliefs regarding their risk of $\mathrm{AD}$ based on family history to be "not new", whereas if test results conflicted with prior beliefs then family history was often more compelling in participants' self-perception of risk. Some participants turned out APOE \&4-negative but continued to feel their actual risk was higher than the risk they learned, because of their family history.

\section{Discussion}

In this review on the psychological, behavioral and social effects of disclosing genetic and nongenetic $\mathrm{AD}$ biomarkers to cognitively healthy research participants we found that disclosing an APOE \&4 genotype to cognitively healthy FDRs of AD patients in a controlled research context does not appear to result in anxiety or depression [23, 29]. Being informed of $\varepsilon 4$-positivity does lead to an increase in testrelated distress, LTC insurance uptake and health-related behavior changes when compared with the disclosure of ع4-negativity, and possibly influences memory functioning [22-24, 29, 32-34]. In addition, in people with a family history of $\mathrm{AD}$, disclosure of APOE genotype does not radically alter beliefs regarding the causation of $\mathrm{AD}$ and personal risk [26]. No studies reporting on the impact of disclosure of nongenetic biomarkers were identified. Only a single study on hypothetical disclosure referred to both genetic and nongenetic biomarkers.

Based on these studies, disclosure of APOE genotype is often considered to result in relief in case of low risk and to be "safe" in case of high risk $[18,36]$. Whereas the first part of this conclusion seems justified [37, 38], several remarks can be made regarding the second.

First, in every study in which genotype was disclosed (except for [32]), participants were FDRs of AD patients. In these individuals the effects of genotype disclosure can be expected to be relatively small, because most may already suspect to be at increased risk on the basis of their family history. Interviews with participants with a FDR with AD show that those with an $\varepsilon 4$ allele are indeed not surprised, whereas those without an $\varepsilon 4$ allele sometimes find it hard to believe [26]. Hence, results from this particular study population cannot simply be generalized to other groups. In research participants who do not have a family history of $\mathrm{AD}$ or who are unaware of the increased risk that is associated with being a FDR of an AD patient, the impact of APOE genotype disclosure may be more substantial. The results of the REVEAL III study, in which a quarter of the participants were not FDRs of AD patients, are expected to show the impact of disclosure in this group and are eagerly awaited [27].

Second, although disclosure does not on average result in more depression, careful interpretation of these results is warranted. In one of the studies, 24 participants (9\%) scored above the clinical cutoff score for depression 12 months after disclosure, while 21 of them scored below this threshold at baseline [31]. In another study, between 15 and $30 \%$ of participants agreed to a statement of feeling depressed after disclosure of an increased $\mathrm{AD}$ risk, whereas none of the low-risk individuals did so [30]. It must be noted that these were all participants who underwent psychological screening prior to inclusion, and that it is currently unknown whether the impact of receiving biomarker results is different in people who already experience psychological problems at baseline. These findings suggest that while the impact of disclosure may be low on average, there may be negative consequences for a subset of participants. Although this subset is relatively small, because $\mathrm{AD}$ research initiatives aim to recruit large numbers of research participants, a considerable number of people could be negatively affected $[5,6]$.

Third, disclosure of an increased genetic risk may negatively influence subjective and objective memory functioning in people without cognitive impairment prior to disclosure [32]. Risk disclosure may thus not 
only lead to a perceived diminishment in memory functioning, but also to a measurable diminishment. A similar effect is known from drug trials in which information on side effects can induce those very symptoms, a phenomenon referred to as the "nocebo" or negative placebo effect. This is supposedly the result of negative expectations regarding the side effects of the intervention [39]. Labeling someone as being at high risk of developing $\mathrm{AD}$ may in the same way create negative expectations of one's own memory functioning, resulting in actual diminished functioning [40].

Fourth, the reported behavior changes after learning one carries an APOE $\varepsilon 4$ allele are not merely positive. On the one hand, the increase in LTC insurance uptake reported by $\varepsilon 4$-positive participants can be considered a benefit because it corresponds to an important reason why people want to know their $\mathrm{AD}$ risk, namely to plan for the future and arrange personal affairs [41-43]. At the same time, fear of insurance discrimination is among the main reasons why people do not want genetic susceptibility testing $[41,42]$. Because the increase in LTC insurance uptake by those at increased risk of developing $\mathrm{AD}$ indicates a potential for adverse selection [24, 34], which insurers may seek to prevent by charging at-risk individuals more or even refusing them as clients, this fear is not farfetched; for instance, current US legislation to protect citizens against insurance discrimination on the basis of genetic information does not apply to LTC and disability insurance [44]. Although seemingly innocent, increasing dietary supplement intake may also have negative consequences. It may give people a false sense of control over their situation, as the most frequently taken supplement after genotype disclosure, vitamin E [33], does not help in preventing $\mathrm{AD}$ [45]. In fact, it may even have a paradoxically negative effect on cognition in some [46] and has significant potential side effects [47].

The conclusion that disclosing increased genetic risk of AD to healthy research participants is "safe" $[18,36]$ thus deserves some nuance. The current findings cannot be extrapolated to nongenetic biomarkers because there are potentially important differences [48]. In particular, the presence of nongenetic biomarkers compatible with an "AD profile", such as a positive amyloid PET scan, is increasingly considered not only as indicative of pathological processes that may eventually lead to the development of $\mathrm{AD}$, but as defining the disease itself, even in the absence of symptoms. In recent research criteria, persons with AD-related nongenetic biomarkers are labeled as being in a "preclinical stage of Alzheimer's disease" [4, 49] suggestive of the inevitable onset of dementia in the future. Although the one study on hypothetical disclosure shows similar envisioned reactions to receiving genetic and nongenetic $\mathrm{AD}$ biomarker results, it also shows that one-third of the respondents do not associate (nongenetic) biomarker evidence of $\mathrm{AD}$ with either the presence of $\mathrm{AD}$ or an increased risk of developing $\mathrm{AD}$ [28]. If people receive nongenetic biomarker results in a research context in which they learn that nongenetic biomarkers are not merely risk factors but reflect an ongoing biological process, their response may strongly differ from their reaction to genetic biomarkers.

\section{Limitations of this systematic review}

The present systematic review does not consider risk perception as a psychological effect in itself and because of that the search strategy does not cover this aspect. Gaining a full understanding of the effects of $\mathrm{AD}$ biomarker disclosure will need to involve further study of how individual risk perception mediates the information disclosed and psychological or behavioral effects. How one perceives one's risk may not always be in line with the risk estimate one received and/or recalls [50]. In that case, instead of mediating, the (wrongly) perceived risk may directly cause psychological effects. Finally, risk perception understood in relation to self-perception is an outcome of interest in itself too. Do participants to whom risk is disclosed see themselves the same as before? Or as people who are destined to develop AD? Or maybe even as people who are already ill, waiting for symptoms to manifest [51]?

A second limitation concerns the risk of bias in individual studies. Although we considered all eligible studies of sufficient quality and none as having a high risk of bias, limited bias could have influenced the results. The included studies do not explicitly mention having excluded people with subjective cognitive complaints. An uneven distribution of people with cognitive complaints over the $\varepsilon 4$-positive and $\varepsilon 4$-negative groups could have interacted with and obscured the relation between the disclosed result and the impact of disclosure. The results of those studies particularly prone to bias (e.g., because of a nonrandomized study design) were and should be interpreted with caution.

Finally, the identified studies had limited heterogeneity, both with regard to the type of biomarker disclosed (APOE genotype only) and with regard to the study population (all US cultural background, mostly FDRs of $\mathrm{AD}$ patients and only people without psychological complaints at baseline). Because studies on the public interest in predictive genetic testing for $\mathrm{AD}$ show significant cultural differences [52], the effects of disclosure may also differ across cultures and settings. Furthermore, because people with moderate to high levels of anxiety, depression and distress at baseline were excluded from the identified studies, the effect of disclosure on these potentially more vulnerable people, is at present unknown. Therefore, one should be careful in extrapolating the results of these studies to other settings and research participant groups. 


\section{Conclusions}

In the context of research, the disclosure of an APOE $\varepsilon 4$ genotype overall has no major psychological, behavioral and social impact on cognitively healthy FDRs of patients with AD. The disclosure does, however, lead to elevated test-related distress, depressive symptoms in some, increased uptake of LTC insurance and changes in health-related behavior such as dietary supplement intake. Furthermore, it may affect subjective and objective memory functioning. The impact of disclosing other, nongenetic, biomarkers such as amyloid PET scans or CSF abnormalities is currently unknown. Knowledge of this impact is needed for research projects to decide on whether, when and how biomarker results should be disclosed. Moreover, it will enable institutional review boards to make informed judgments of the risk-benefit ratios in proposed studies, and potential participants in choosing whether or not to participate. Therefore, before engaging in large-scale research projects disclosing biomarkers other than APOE genotype to persons without a family history of $\mathrm{AD}$, research on the psychological, behavioral and social impact of this disclosure is indispensable.

\section{Additional files}

\section{Additional file 1: Includes the full search strategy per electronic} database. (DOCX $15 \mathrm{~kb}$ )

Additional file 2: A table presenting the data extraction form used for quality assessment of the included studies and data synthesis. (DOCX $14 \mathrm{~kb}$ )

Additional file 3: A table presenting the potential for bias in individual studies via an assessment of main potential sources and types of bias per study included in the systematic review. (DOCX $18 \mathrm{~kb}$ )

\section{Abbreviations}

AD: Alzheimer's disease; APOE: Apolipoprotein E; BAl: Beck Anxiety Inventory; CES-D: Center for Epidemiologic Studies Depression Scale; CSF: Cerebrospinal fluid; $\varepsilon 4$ : Apolipoprotein E $\varepsilon 4$ allele; FDR: First-degree relative; IES: Impact of Event Scale; LTC: Long-term care; MFQ: Memory Functioning Questionnaire; MIA: Metamemory in Adulthood; PET: Positron emission tomography; REVEAL: Risk Evaluation and Education for Alzheimer's Disease Study; ROCFT: Rey-Osterrieth Complex Figure Test; WMS-R: Wechsler Memory Scale Revised

\section{Acknowledgements}

The authors thank Wichor Bramer, information specialist at the Erasmus Medical Center library, for his contribution in generating search strategies for all electronic databases.

\section{Funding}

This work was performed within the European Prevention of Alzheimer's Dementia consortium (EPAD), which is funded by the Innovative Medicines Initiative under grant agreement 115736. The research question of this systematic review arose within the ethical work package providing guidance to the EPAD project, an AD prevention study that will soon recruit large numbers of cognitively healthy participants on which biomarker examinations will be performed. The design of the review, data collection, analysis and interpretation were not influenced by the funding body, neither was the writing of the manuscript. SB and CB receive funding from the Cambridge NIHR Biomedical Research Centre for related work.

\section{Availability of data and materials}

All conclusions drawn are based on published data from included studies that are presented in Table 2 of this article and can be retrieved by searching the original articles. The protocol of this systematic review is available upon request.

\section{Authors' contributions}

SASAB participated in the design of the review, the development of the search strategies and screening and selection of eligible articles, carried out the critical appraisal and data extraction, and was involved in data synthesis, data interpretation and writing the manuscript. KT contributed to the design of the review and the development of search strategies, and participated in screening and selection of eligible articles, data synthesis and interpretation, and in writing the manuscript. EMB contributed to the design of the review, screening and selection of eligible articles, data synthesis and interpretation, and writing the manuscript. RJM contributed to the design of the review and interpretation of the data and revised the manuscript. SB contributed to the design of the review and interpretation of the data and revised the manuscript. CB contributed to the conception and design of the review, interpretation of the data and critical revision of the manuscript. MHS contributed to the design of the review and interpretation of the data and revised the manuscript. ER guided the design of the review, the data acquisition, analysis, synthesis and interpretation and writing of the manuscript. All authors approved the manuscript.

\section{Authors' information}

Not applicable.

\section{Competing interests}

The authors declare that they have no competing interests.

\section{Consent for publication}

Not applicable.

Ethics approval and consent to participate

Not applicable.

\section{Author details}

'Department of Neurology, Radboudumc, Geert Grooteplein-Zuid 10, 6525 GA Nijmegen, The Netherlands. ${ }^{2}$ Department of Medical Ethics and Philosophy of Medicine, Erasmus MC, Wytemaweg 80, 3015 CN Rotterdam, The Netherlands. ${ }^{3}$ Cambridge Institute of Public Health, University of Cambridge, Forvie Site, Robinson Way, Cambridge CB2 OSR, UK.

Received: 29 May 2016 Accepted: 4 October 2016

Published online: 10 November 2016

References

1. Daviglus ML, Plassman BL, Pirzada A, Bell CC, Bowen PE, Burke JR, et al. Risk factors and preventive interventions for Alzheimer disease: state of the science. Arch Neurol. 2011;68:1185-90.

2. Bateman RJ, Xiong C, Benzinger TL, Fagan AM, Goate A, Fox NC, et al. Clinical and biomarker changes in dominantly inherited Alzheimer's disease. N Engl J Med. 2012;367:795-804.

3. Villemagne VL, Burnham S, Bourgeat P, Brown B, Ellis KA, Salvado O, et al. Amyloid beta deposition, neurodegeneration, and cognitive decline in sporadic Alzheimer's disease: a prospective cohort study. Lancet Neurol. 2013;12:357-67.

4. Sperling RA, Aisen PS, Beckett LA, Bennett DA, Craft S, Fagan AM, et al. Toward defining the preclinical stages of Alzheimer's disease: recommendations from the National Institute on Aging-Alzheimer's Association workgroups on diagnostic guidelines for Alzheimer's disease. Alzheimers Dement. 2011;7:280-92.

5. Sperling RA, Rentz DM, Johnson KA, Karlawish J, Donohue M, Salmon DP, et al. The A4 study: stopping AD before symptoms begin? Sci Transl Med. 2014;6:228fs 13 .

6. Ritchie CW, Molinuevo JL, Truyen L, Satlin A, Van der Geyten S, Lovestone S, et al. Development of interventions for the secondary prevention of Alzheimer's dementia: the European Prevention of Alzheimer's Dementia (EPAD) project. Lancet Psychiatry. 2016;3:179-86. 
7. Jack CR, Knopman DS, Jagust WJ, Shaw LM, Aisen PS, Weiner MW, et al. Hypothetical model of dynamic biomarkers of the Alzheimer's pathological cascade. Lancet Neurol. 2010;9:119-28.

8. Rowe CC, Ellis KA, Rimajova M, Bourgeat P, Pike KE, Jones G, et al. Amyloid imaging results from the Australian Imaging, Biomarkers and Lifestyle (AIBL) study of aging. Neurobiol Aging. 2010;31:1275-83.

9. Cupples LA, Farrer LA, Sadovnick AD, Relkin N, Whitehouse P, Green RC. Estimating risk curves for first-degree relatives of patients with Alzheimer's disease: the REVEAL study. Genet Med. 2004;6:192-6.

10. Alzheimer Europe. http://www.alzheimer-europe.org/Research/Value-ofKnowing/Public-concerns-about-Alzheimer-s-disease. Accessed 24 Apr 2016.

11. Roberts JS, Connell CM. Illness representations among first-degree relatives of people with Alzheimer disease. Alzheimer Dis Assoc Disord. 2000;14:129-36.

12. Morris JC, Roe CM, Grant EA, Head D, Storandt M, Goate AM, et al. Pittsburgh compound $B$ imaging and prediction of progression from cognitive normality to symptomatic Alzheimer disease. Arch Neurol. 2009:66:1469-75.

13. Farrer LA, Cupples LA, Haines JL, Hyman B, Kukull WA, Mayeux R, et al. Effects of age, sex, and ethnicity on the association between apolipoprotein E genotype and Alzheimer disease. A meta-analysis. APOE and Alzheimer Disease Meta Analysis Consortium. JAMA. 1997;278:1349-56.

14. Grill JD, Johnson DK, Burns JM. Should we disclose amyloid imaging results to cognitively normal individuals? Neurodegener Dis Manag. 2013;3:43-51.

15. Aizenstein HJ, Nebes RD, Saxton JA, Price JC, Mathis CA, Tsopelas ND, et al. Frequent amyloid deposition without significant cognitive impairment among the elderly. Arch Neurol. 2008;65:1509-17.

16. Mathis CA, Kuller LH, Klunk WE, Snitz BE, Price JC, Weissfeld LA, et al. In vivo assessment of amyloid-beta deposition in nondemented very elderly subjects. Ann Neurol. 2013;73:751-61.

17. Heshka JT, Palleschi C, Howley H, Wilson B, Wells PS. A systematic review of perceived risks, psychological and behavioral impacts of genetic testing. Genet Med. 2008;10:19-32.

18. Rahman B, Meiser B, Sachdev P, Barlow-Stewart K, Otlowski M, Zilliacus E, et al. To know or not to know: an update of the literature on the psychological and behavioral impact of genetic testing for Alzheimer disease risk. Genet Test Mol Biomarkers. 2012;16:935-42.

19. Moher D, Liberati A, Tetzlaff J, Altman DG, Group P. Preferred reporting items for systematic reviews and meta-analyses: the PRISMA statement. PLoS Med. 2009;6:e1000097.

20. Higgins JP, Altman DG, Gotzsche PC, Jüni $P$, Moher D, Oxman AD, et al. The Cochrane Collaboration's tool for assessing risk of bias in randomised trials. BMJ. 2011;343:d5928.

21. Critical Appraisal Skills Programme (CASP). http://www.casp-uk.net/ checklists. Accessed 27 Jan 2016.

22. Chao S, Roberts JS, Marteau TM, Silliman R, Cupples LA, Green RC. Health behavior changes after genetic risk assessment for Alzheimer disease: the REVEAL study. Alzheimer Dis Assoc Disord. 2008;22:94-7.

23. Green RC, Roberts JS, Cupples LA, Relkin NR, Whitehouse PJ, Brown T, et al. Disclosure of APOE genotype for risk of Alzheimer's disease. N Engl J Med. 2009;361:245-54

24. Zick CD, Mathews CJ, Roberts JS, Cook-Deegan R, Pokorski RJ, Green RC. Genetic testing for Alzheimer's disease and its impact on insurance purchasing behavior. Health Aff (Millwood). 2005;24:483-90

25. Gooding HC, Linnenbringer EL, Burack J, Roberts JS, Green RC, Biesecker BB. Genetic susceptibility testing for Alzheimer disease: motivation to obtain information and control as precursors to coping with increased risk. Patient Educ Couns. 2006;64:259-67.

26. Chilibeck G, Lock M, Sehdev M. Postgenomics, uncertain futures, and the familiarization of susceptibility genes. Soc Sci Med. 2011;72:1768-75.

27. Christensen KD, Roberts JS, Zikmund-Fisher BJ, Kardia SL, McBride CM Linnenbringer $E$, et al. Associations between self-referral and health behavior responses to genetic risk information. Genome Med. 2015;7:10.

28. Caselli RJ, Langbaum J, Marchant GE, Lindor RA, Hunt KS, Henslin BR, et al. Public perceptions of presymptomatic testing for Alzheimer disease. Mayo Clin Proc. 2014;89:1389-96.

29. Green RC, Christensen KD, Cupples LA, Relkin NR, Whitehouse PJ, Royal CDM, et al. A randomized noninferiority trial of condensed protocols for genetic risk disclosure of Alzheimer's disease. Alzheimers Dement. 2014;11:1222-30.

30. Romero L, Garry PJ, Schuyler M, Bennahum DA, Qualls C, Ballinger L, et al. Emotional responses to APO E genotype disclosure for Alzheimer disease. J Genet Couns. 2005;14:141-50.
31. Ashida S, Koehly LM, Roberts JS, Chen CA, Hiraki S, Green RC. The role of disease perceptions and results sharing in psychological adaptation after genetic susceptibility testing: the REVEAL study. Eur J Hum Genet. 2010;18:1296-301.

32. Lineweaver TT, Bondi MW, Galasko D, Salmon DP. Effect of knowledge of APOE genotype on subjective and objective memory performance in healthy older adults. Am J Psychiatry. 2014;171:201-8.

33. Vernarelli JA, Roberts JS, Hiraki S, Chen CA, Cupples LA, Green RC. Effect of Alzheimer disease genetic risk disclosure on dietary supplement use. Am J Clin Nutr. 2010;91:1402-7.

34. Taylor DH, Cook-Deegan RM, Hiraki S, Roberts JS, Blazer DG, Green RC. Genetic testing for Alzheimer's and long-term care insurance. Health Aff (Milwood). 2010;29:102-8.

35. Ashida S, Koehly LM, Roberts JS, Chen CA, Hiraki S, Green RC. Disclosing the disclosure: factors associated with communicating the results of genetic susceptibility testing for Alzheimer's disease. J Health Commun. 2009;14:768-84.

36. Arribas-Ayllon M. The ethics of disclosing genetic diagnosis for Alzheimer's disease: do we need a new paradigm? Br Med Bull. 2011;100:7-21.

37. LaRusse S, Roberts JS, Marteau TM, Katzen H. Genetic susceptibility testing versus family history-based risk assessment: Impact on perceived risk of Alzheimer disease. Genet Med. 2005;7:48-53.

38. Marteau TM, Roberts S, LaRusse S, Green RC. Predictive genetic testing for Alzheimer's diseases: Impact upon risk perception. Risk Anal. 2005;25:397-404.

39. Wells RE, Kaptchuk TJ. To tell the truth, the whole truth, may do patients harm: the problem of the nocebo effect for informed consent. Am J Bioeth. 2012;12:22-9.

40. Symon A, Williams B, Adelasoye QA, Cheyne H. Nocebo and the potential harm of "high risk" labelling: a scoping review. J Adv Nurs. 2015;71:1518-29.

41. Cutler SJ, Hodgson LG. To test or not to test: interest in genetic testing for Alzheimer's disease among middle-aged adults. Am J Alzheimers Dis Other Demen. 2003;18:9-20.

42. Roberts JS. Anticipating response to predictive genetic testing for Alzheimer's disease: a survey of first-degree relatives. Gerontologist. 2000;40:43-52.

43. Roberts JS, LaRusse SA, Katzen H, Whitehouse PJ, Barber M, Post SG, et al. Reasons for seeking genetic susceptibility testing among first-degree relatives of people with Alzheimer disease. Alzheimer Dis Assoc Disord. 2003;17:86-93.

44. Arias JJ, Karlawish J. Confidentiality in preclinical Alzheimer disease studies: when research and medical records meet. Neurology. 2014;82:725-9.

45. Morris MC, Evans DA, Bienias JL, Tangney CC, Bennett DA, Aggarwal N, et al Dietary intake of antioxidant nutrients and the risk of incident Alzheimer disease in a biracial community study. JAMA. 2002;287:3230-7.

46. Lloret A, Badia MC, Mora NJ, Pallardo FV, Alonso MD, Vina J. Vitamin E paradox in Alzheimer's disease: it does not prevent loss of cognition and may even be detrimental. J Alzheimers Dis. 2009;17:143-9.

47. Miller ER, Pastor-Barriuso R, Dalal D, Riemersma RA, Appel LJ, Guallar E. Meta-analysis: high-dosage vitamin E supplementation may increase allcause mortality. Ann Intern Med. 2005;142:37-46.

48. Roberts JS, Dunn LB, Rabinovici GD. Amyloid imaging, risk disclosure and Alzheimer's disease: ethical and practical issues. Neurodegener Dis Manag. 2013;3:219-29.

49. Dubois B, Feldman HH, Jacova C, Cummings $\mathrm{J}$, Dekosky ST, BarbergerGateau P, et al. Revising the definition of Alzheimer's disease: a new lexicon. Lancet Neurol. 2010;9:1118-27.

50. Linnenbringer E, Roberts JS, Hiraki S, Cupples LA, Green RC. "I know what you told me, but this is what I think:" perceived risk of Alzheimer disease among individuals who accurately recall their genetics-based risk estimate. Genet Med. 2010;12:219-27.

51. Timmermans S, Buchbinder M. Patients-in-waiting: Living between sickness and health in the genomics era. J Health Soc Behav. 2010;51:408-23.

52. Wikler EM, Blendon RJ, Benson JM. Would you want to know? Public attitudes on early diagnostic testing for Alzheimer's disease. Alzheimers Res Ther. 2013;5:43.

53. Fanshawe TR, Prevost AT, Roberts JS, Green RC, Armstrong D, Marteau TM. Explaining behavior change after genetic testing: the problem of collinearity between test results and risk estimates. Genet Test. 2008;12:381-6.

54. Cassidy MR, Roberts JS, Bird TD, Steinbart EJ, Cupples LA, Chen CA, et al. Comparing test-specific distress of susceptibility versus deterministic genetic testing for Alzheimer's disease. Alzheimers Dement. 2008;4:406-13.

55. Roberts JS, Cupples LA, Relkin NR, Whitehouse PJ, Green RC, Group RS. Genetic risk assessment for adult children of people with Alzheimer's disease: the Risk Evaluation and Education for Alzheimer's Disease (REVEAL) study. J Geriatr Psychiatry Neurol. 2005;18:250-55. 